\title{
The influence of Ac parameters in the process of micro-arc oxidation film electric breakdown
}

\author{
Jin MA \\ Institute of Mechanical and Electronic Engineering, Wuhan University of Technology, Wuhan, China, 430070
}

\begin{abstract}
This paper studies the electric breakdown discharge process of micro-arc oxidation film on the surface of aluminum alloy. Based on the analysis of the AC parameters variation in the micro-arc oxidation process, the following conclusions can be drawn: The growth of oxide film can be divided into three stages, and Oxide film breakdown discharge occurs twice in the micro-arc oxidation process. The first stage is the formation and disruptive discharge of amorphous oxide film, producing the ceramic oxide granules, which belong to solid dielectric breakdown. In this stage the membrane voltage of the oxide film plays a key role; the second stage is the formation of ceramic oxide film, the ceramic oxide granules turns into porous structure oxide film in this stage; the third stage is the growth of ceramic oxide film, the gas film that forms in the oxide film's porous structure is electric broken-down, which is the second breakdown discharge process, the current density on the oxide film surface could affect the breakdown process significantly.
\end{abstract}

Keywords: micro-arc oxidation; AC parameter; solid dielectric; gas film; break down

\section{Introduction}

Micro-arc oxidation, also called micro-plasma oxidation, is a technique to grow ceramic oxide films in micro areas with high temperature environment through complex electrochemical, plasma-chemical and thermochemical processes caused by the micro plasma discharging on the light alloy surface. The ceramic films have some unique characteristics such as strong adhesive strength with substrate, good electrical insulation, corrosion resistance and high hardness, which can largely enhance the properties of alloy surface ${ }^{[1]}$. In order to study the influence of AC parameters on the oxide film breakdown process systematically and quantitatively, the test of micro-arc oxidation on the aluminum alloy is carried out by applying AC power in weak alkaline electrolyte in this paper.

\section{Material and Equipment}

The alloy material for experimentation is $2 \mathrm{~A} 12$ aluminum alloy which has been made into cuboid samples with the size of $14 \times 8 \times 2 \mathrm{~mm}$. The top $4 \mathrm{~mm}$ of the sample is the wire connection area which is covered by insulating material. The area of sample surface contacting with the electrolyte aqueous solution is $2.16 \mathrm{~cm}^{2}$.

The micro-arc oxidation device is homemade with an AC output voltage ranging from 0 to $700 \mathrm{~V}$ (step-less voltage regulation) with $50 \mathrm{~Hz}$ frequency. As a result of

${ }^{a}$ Corresponding author: matinjin@whut.edu.cn
AC power, the two $\mathrm{AC}$ electrodes sample electrode (a) and stainless steel electrode (c) are cathode and anode mutually. An auxiliary inert silver electrode (b) is fastened $1 \mathrm{~mm}$ away from the sample surface. The electrolyte solution is alkalescency electrolyte solution. Homemade cooling device is installed in the cooling bay to keep the temperature of electrolyte aqueous solution under $25^{\circ} \mathrm{C}$. The electrolyte can keep flowing by using a water pump.

\section{Method and Process}

Samples with the same specification are disposed by micro-arc oxidation test, using AC power source in the electrolyte solution which has the same ratio of solubility. The initial AC current is at $1 \mathrm{~A}$. Experimental results are measured and plotted as shown in Fig 1 and Fig 2.

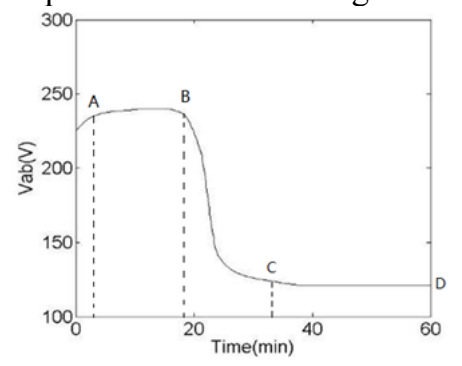

Figure 1.Variation curve of Vab in micro-arc oxidation process 


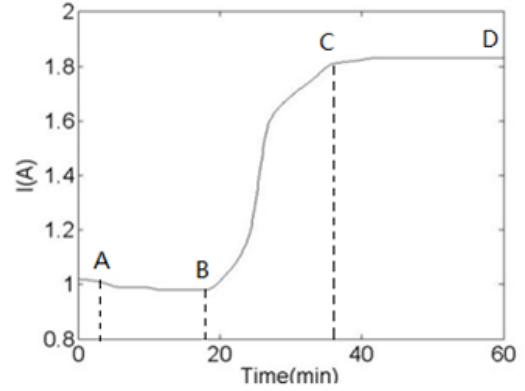

Figure 2.Variation curve of I in micro-arc oxidation process

According to the Fig.1 and Fig.2, it can be judged that the point $\mathrm{A}$ and $\mathrm{B}$ is the important time nodes in the process of micro-arc oxidation.

The disposing time for samples is $10 \mathrm{sec}, 5 \mathrm{~min}, 20$ min and $40 \mathrm{~min}$ respectively. After the micro-arc oxidation treatment, measure the thickness of samples surface oxide film, analyze the morphology of oxide films.

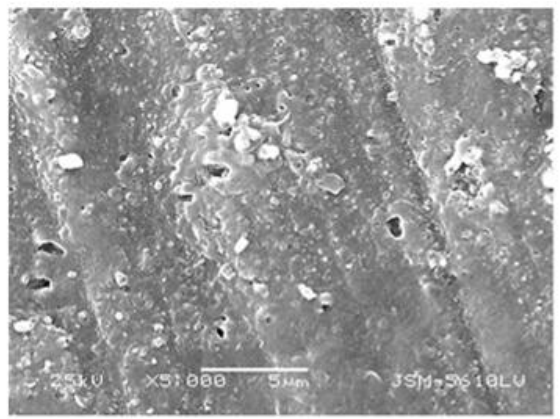

Figure 3.Morphology of oxide film at $10 \mathrm{sec}$

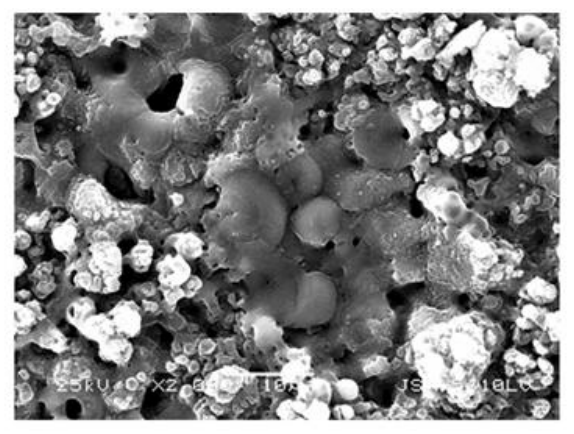

Figure 4.Morphology of oxide film at $5 \mathrm{~min}$

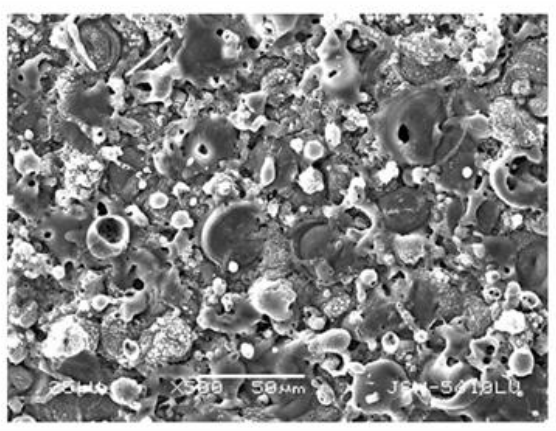

Figure 5.Morphology of oxide film at $20 \mathrm{~min}$

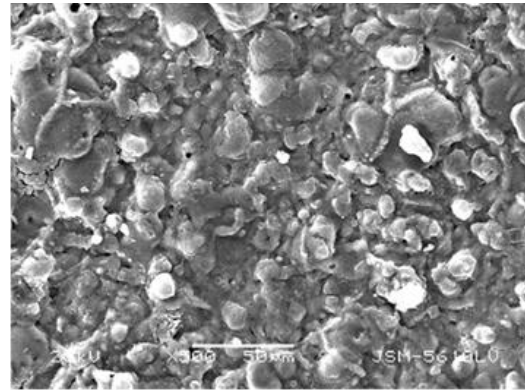

Figure 6.Morphology of oxide film at $40 \mathrm{~min}$

It can be seen from Fig. 3 that a thin oxide film has formed at $10 \mathrm{sec}$ of the micro-arc oxidation process with separate oxide granules in size of appr 1 um preliminary occurred on it. According to Fig.4, when oxidation time extended to $5 \mathrm{~min}$, the number of oxide granules with the average size appr. 5um gradually increased and started mutual cross-linking to cover the sample surface. After $20 \mathrm{~min}$, as is shown in Fig.5, the size of granules was appr. $10 \mathrm{um}$, with granules becoming mutual superposed and forming a porous film structure. As can be seen from Fig.6, granules fused mutually and no obvious boundaries remained at $40 \mathrm{~min}$, crater shape hole was formed with the size of appr.10 um.

\section{Results and Discussion}

According to the experimental phenomenon and the variation of electrical parameters in the process of microarc oxidation, the growth mechanism of micro-arc oxidation film can be divided into three stages.

\subsection{Stage 1 Forming of amorphous oxide film}

On the sample surface, the Al element got rapid electrochemical reactions when the sample was put into electrolyte and start charging, a thin amorphous oxide film with the thickness less than 1um got rapidly formed ${ }^{[2]}$. After the film was formed, charge accumulates on both sides of the film under the effect of electric field, thus forming a capacitor ${ }^{[3]}$. In the electrolyte side of the amorphous oxide film, a certain concentration gradient of charge is accumulated under the effect of electric field ${ }^{[4]}$; with the ignorance of the concentration between auxiliary electrode and the oxidation film, $\mathrm{V}_{\mathrm{ab}}$ can be regarded as the voltage between amorphous oxide film. When the charge accumulates till $\mathrm{V}_{\mathrm{ab}}$ reaches the breakdown voltage of amorphous oxide film, the weakest part would be punctured so the micro-arc discharging phenomenon emerges, which produces high temperature and high pressure, oxidizing Al into ceramic oxide granules (as is shown in Fig.3). This is the first breakdown discharge process. Due to micro-arc discharging, the accumulated electric quantity on the surface of amorphous oxide film decreased. Under the effect of electric field, the charged ions in the electrolyte migrate as a supplementary of micro-arc electric consuming. When the electric quantity of consumption and supplementary reaches a dynamic equilibrium, $\mathrm{V}_{\mathrm{ab}}$ remains relatively stable.

When the first electric breakdown occurs, $V_{a b}$ values tend to be the limit value $183 \mathrm{v}$, which means the critical breakdown voltage of oxide film is $183 \mathrm{~V}$. According to 
the formula of critical breakdown field strength ${ }^{[5]}$, oxide film critical breakdown field strength can be estimated by Eq. (1):

$$
E_{b}=\sqrt{2} V / b
$$

Where $\mathrm{E}_{\mathrm{b}}=2588 \mathrm{KV} / \mathrm{cm}$ and $\mathrm{b}$ is thickness of oxide film.

As the value of solid dielectric electric breakdown field strength is about $10^{4}-10^{6} \mathrm{~V} / \mathrm{cm}$ orders of magnitude, and the critical breakdown field strength of amorphous oxide film is within the scope of this order of magnitude, so the breakdown of amorphous oxide film should belong to solid dielectric breakdown.

In the process of oxidation film breakdown for the first time, membrane voltage of oxide film plays a key role. Specifically, membrane voltage must be higher than the critical breakdown voltage of oxide film so that the oxide film can be breakdown.

\subsection{Stage 2 Forming of the ceramic oxide film.}

The ceramic oxide granules formed by micro-arc discharging strengthened the weak part of initial oxide film. As a result, accumulation in both sides of amorphous oxide film continually increases, punctured and strengthened the weak part of film by ceramic oxide granule constantly. As a consequence, $\mathrm{V}_{\mathrm{ab}}$ increased gradually at the forming stage of ceramic oxide film (in the period A to B as shown in Fig.1).With the proceeding of micro-arc oxidation, the granules increased and crosslinked gradually (as shown in Fig.4), forming porous structure of ceramic oxide film by cover the sample surface. The thickness of film is about 5-10 um at this stage.

\subsection{Stage 3 Growth of ceramic oxide film.}

The ceramic oxide granules gradually formed porous structure of ceramic oxide film. Due to the electrolytic reaction, the gas film in the pore space is formed when oxygen precipitated from anode and emerges from the porous structure of ceramic oxide film, as shown in Fig.7.

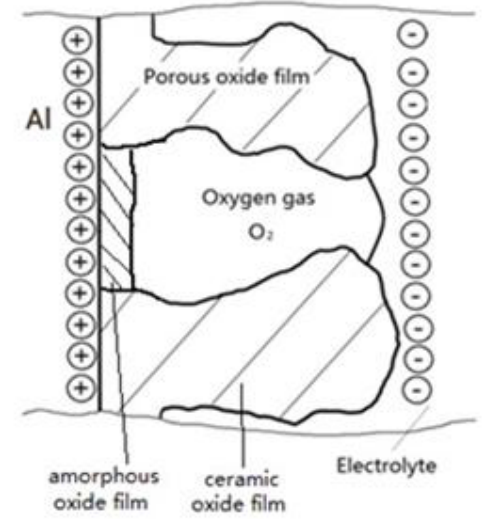

Figure 7.Oxygen gas film forming in the pore of porous structure

The breakdown field intensity of oxygen is about $30 \mathrm{KV} / \mathrm{cm}^{[5]}$ and the thickness of oxide film is about 10um. Therefore the gas film would be broken down when the voltage between both sides is above $30 \mathrm{~V}$, and then micro-arc discharges. This is the second breakdown discharge process. The gaseous dielectric breakdown is easier other than the solid dielectric breakdown at the first stage. Under the joule heating effect, micro-arc discharge released a lot of heat in the pore, forming a high temperature and high pressure micro area to oxidate Al into high temperature molten oxides. The molten oxides expanded and blend with each other with continuous growth under high pressure, as is shown in Fig.5. At this stage, the growth rate of oxide film increased rapidly.

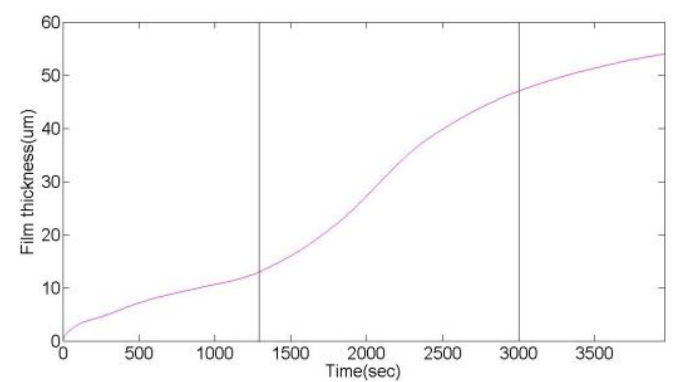

Figure 8. The thickness curve of oxide film in micro-arc oxidation process

According to Fig 8, at stage 1 and stage 2, oxide film growth is very slowly. At stage 3 the growth of oxide film becomes quickly.

Because of rapid discharging of gas film breakdown, supplementary electric should be obtained from electrolyte outside the pores. However, the migration speed of charged ions in electrolyte cannot compensate the consuming electric quantity due to the limitation of the electrolyte conductivity. As a consequence, Vab value decreased rapidly and current value increased rapidly (in the period $\mathrm{B}$ to $\mathrm{C}$ as is shown in Fig.1 and Fig.2.).

Along with the oxidation film growing, the thickness of film is increasing while electric field intensity between film sides is gradually weakening. When electric field intensity is unable to breakdown the gas film, the microarc stopped discharging and the film's thickness reached a max-value.

By keeping the voltage between electrode (a) and electrode (c) at $600 \mathrm{~V}$, different initial current densities $(10 \mathrm{~A} / \mathrm{dm} 2 、 20 \mathrm{~A} / \mathrm{dm} 2 、 30 \mathrm{~A} / \mathrm{dm} 2 、 40 \mathrm{~A} / \mathrm{dm} 2 、 50 \mathrm{~A} / \mathrm{dm} 2$ 、 $60 \mathrm{~A} / \mathrm{dm} 2$ ) are employed to carry on micro-arc oxidation experiments. Variation of current is measured in the process of micro-arc oxidation test and drawn as shown in Fig 9.

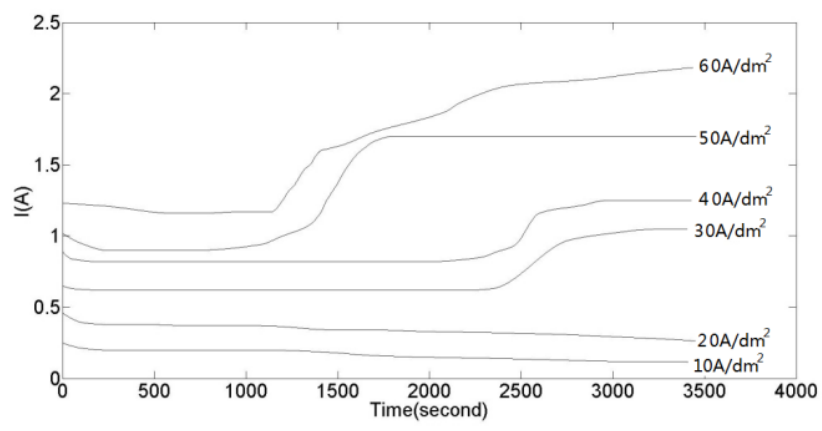

Figure 9.Variation of current in micro-arc oxidation process by different initial current density

From the figure 8, which shows the process of microarc oxidation, when the initial current density is less than $30 \mathrm{~A} / \mathrm{dm}^{2}$, the second breakdown does not occur. This is 
due to the liquid - gas interface formation between the electrolyte and the gas, charged particles must have sufficient kinetic energy to pass through the liquid - gas interface. With enough energy, it rushes into gas film and generates gas ionization that cause gas film electric breakdown. With too low current density, the charged particles in the electrolyte move too slow, which would decrease the gas film breakdown probability. So the second breakdown cannot occur on the sample surface, and the growth of the ceramic oxide film is in the stagnation.

\section{Conclusions}

Based on the analysis and discussion, the following conclusions can be drawn:

1)The growth of ceramic oxide film can be divided into three stages: the formation and breakdown stage for amorphous oxide film; the formation stage for porous ceramic oxide film and the growth stage for ceramic oxide film.

2) In the first stage, amorphous oxide film forms quickly at the test beginning, the ceramic oxide granules is produced as a result of oxide film's electric breakdown discharge. This is the first breakdown discharge process which belongs to solid dielectric breakdown. Membrane voltage must be higher than the critical breakdown voltage of oxide film to enable the oxide film to be breakdown.

3) In the second stage, ceramic oxide granules gradually grow up into porous structure of ceramic oxide film.

4) In the third stage, oxygen gas film is formed in the pores of ceramic oxide film. Due to the gas film's breakdown, the micro-arc discharge release large amounts of heat to form micro area of high temperature and high pressure, which provide the condition for ceramic oxide film's continuous growth. This is the second breakdown discharge process which belongs to gaseous dielectric breakdown. In this stage, initial current density must be higher than $30 \mathrm{~A} / \mathrm{dm}^{2}$ to make the second breakdown occur, and the ceramic oxide film can growth rapidly.

\section{References}

1. Hu.Zhenqian, Ma.Jin, Heat Treatment of Metals, 28, 23-26 (2003)

2. Ma.Jin, Hu.Zhenqian, Material\&Heat Treatment, 41, 142-144 (2012)

3. Jia.Zheng, Dai.Changsong,Chen.Ling, Measurement Methods of Electrochemistry, Chemical Industry Press, (2006)

4. W. Plieth, Electrochemistry for Materials Science, Science Press, (2008)

5. Yin. Zhiwen, Dielectric Physics, Science Press, (2006)

6. Ma.Jin, ICMME 2014, Applied Mechanics and Materials, 697, 7-11(2015)
7. Vangolu. Y, Alsaran. A, Yildirim.O.S, Wear, 271, 2322-2327(2011)

8. Jacob.Roshan, Srinivasan. S.A, Sivaprasad. K, Muthupandi. V, Materials Science Forum, 765,634-638(2013)

9. Shin. Sowoon, Choi. Yong-Wook, Choi.Jinsub, Materials Letters, 105, 117-119(2013)

10. Tao.Hong, Ho.Soking, Li.echo, Zhang. Xuezhu, Advanced Materials Research, 905, 146$150(2014)$

11. Dey, Arjun, Rani. R.Uma, Thota. Hari Krishna, 39, 3313-3320(2013)

12. Urbahs. A, Urbaha. M, Zujevs. V, Savkovs, K, Rijkuris, G, Transport Means - Proceedings of the International Conference, 101-105(2013)

13. Durdu.Salih, Aytaç.Aylin, Usta.Metin, Journal of Alloys and Compounds, 509, 8601-8606 (2011)

14. Yavuz.H.G, Gunyuz.M, Ozkara.I.M, Baydogan. M, Cimenoglu.H, Defect and Diffusion Forum, 312-315, 877-88(2011)

15. Ma.Jin, Journal of Materials Protection, 47, 4143(2014) 